\title{
Pharmacological Management of Allergic Rhinitis in the Elderly
}

\author{
Andrzej Bozek ${ }^{1}$
}

Published online: 3 December 2016

(C) The Author(s) 2016. This article is published with open access at Springerlink.com

\begin{abstract}
Recent studies suggest that allergic rhinitis is highly prevalent in the elderly population, but is underdiagnosed and undertreated. This review article briefly introduces allergic rhinitis in the elderly (epidemiology and pathophysiology) and identifies the main goals of treatment in these patients with respect to age-related physiological factors, comorbid conditions and polypharmacy. The primary focus of the article is a narrative review of the literature concerning the different types of treatment options in elderly patients aged 60+ years (pharmacological therapy and allergen-specific immunotherapy). The main management trend for allergic rhinitis in elderly patients is the same as the trend in young patients. Second-generation antihistamines and nasal glucocorticosteroids are also the first-line therapies in seniors. In a few trials, allergenspecific immunotherapy for grass pollen or house dust mites has been shown to be effective and safe in patients aged 60 years or older with allergic rhinitis. In conclusion, undertreatment of allergic rhinitis in the elderly is a reality. Pharmacological treatment is quite similar in both older and younger patients with allergic rhinitis.
\end{abstract}

Andrzej Bozek

andrzejbozek@o2.pl

1 Clinical Department of Internal Disease, Dermatology and Allergology, Medical University of Silesia in Katowice, MC Sklodowskiej 10, 41-800 Zabrze, Poland

\section{Key Points}

Allergic rhinitis is undertreated in elderly patients.

Antihistamines and nasal glucocorticosteroids are the first-line therapies in patients over 60 years of age.

Attention should be paid to the use of oral antihistamines in patients with comorbidities and polymedication.

\section{Introduction}

Allergic rhinitis is a significant problem worldwide. The prevalence of rhinitis in the USA is estimated to be between 10 and $30 \%$ in adults [1, 2]. According to Allergic Rhinitis and its Impact on Asthma (ARIA), the prevalence of allergic rhinitis in the population in general in Europe and the USA is between 40 and 50\% [1]. Unfortunately, the ARIA guidelines are not focused on seniors and do not evaluate differences in the diagnosis and treatment of rhinitis. ARIA and the Joint Task Force highlight the problem of rhinitis in the elderly, but do not link rhinitis to allergies [1, 3]. No data are available concerning how allergic rhinitis affects the quality of life of elderly patients and how to determine the needs of elderly people with allergic rhinitis $[2,3]$.

The increasing number of patients with allergies and the aging world population are causing an increase in allergic diseases in patients over 60 years of age $[4,5]$. Although many authors have analyzed the epidemiology, diagnosis and treatment of allergies, the occurrence and natural 
course of allergic disease in elderly patients have been somewhat neglected. The prevalence of allergic rhinitis in elderly patients is approximately $5-8 \%$ and decreases with age [6-8], although some national epidemiological data suggest that the number of patients with allergic diseases may be greater than $10 \%[9,10]$. However, the available epidemiological data are rudimentary, and international cohort studies based on a large elderly population are lacking. Therefore, allergic rhinitis in elderly people remains poorly perceived [10-13].

Few studies have analyzed groups of patients over 60 years of age. Some studies have analyzed the epidemiological situation of rhinitis in adults or elderly people without considering allergic mechanisms.

Various types of chronic rhinitis that sometimes impede a proper diagnosis are noticeable in elderly people $[4,11]$. In addition to allergic rhinitis, non-allergic types of rhinitis include vasomotoric rhinitis, atrophic rhinitis, drug-induced rhinitis and non-allergic rhinitis with eosinophilia [11].

The aim of this article is to review the present literature on allergic rhinitis in the elderly and to discuss the approach to treatment in these patients, including pharmacotherapy and allergen-specific immunotherapy. A cutoff age of 60 years was used as this returned the largest number of studies that discussed allergic rhinitis in the elderly. However, the age limit that defines "elderly" is variable and a trend toward a change to 65,70 years or even higher has been observed.

\section{Aging of the Nose and Immune System}

\subsection{Aging of the Nose}

Nasal anatomical and physiological changes during aging significantly influence rhinitis symptoms. Aging causes an increase in cholinergic activity and mucosal atrophy, decreased mucosal elasticity, weakened mucociliary functions and weakening of the septal cartilage [2, 14]. The nasal mucosa becomes dry and nasal congestion is frequent $[14,15]$. Nasal congestion leads to irritation and damage of the mucosa and causes epistaxis [8]. Toppozada suggested that the nasal mucosa was normal, although the basement membrane became thicker and structural atrophy was present, in postmenopausal subjects [16]. However, other studies did not confirm these changes [17].

\subsection{Immunosenescence}

The aging immune system causes many changes, including a decreased non-specific immune response with a decline in the activity of phagocytes and cytotoxic cells. Significant changes have been observed in $\mathrm{T}$ lymphocyte profiles during aging, including a shift toward CD4+ T memory cells and a reduction in naive $\mathrm{T}$ lymphocytes $[8,18]$. Additionally, a decrease occurs in the proliferative $\mathrm{T}$ lymphocyte response and the cytokine production profile shifts from Th1 to Th2. A decrease in the number and activity of B lymphocytes and the presence of higher concentrations of autoantibodies in the serum are typical of the humoral immune response in elderly patients $[18,19]$. This "immunosenescence" is a result of natural genetic aging, environmental factors and lifestyle [8,9]. However, these changes do not appear to significantly limit the prevalence of allergic diseases in elderly patients [8]. The onset of immunosenescence is very individual, but is observed in all patients over 75 years of age. However, this phenomenon is often not noticeable in individuals between 60 and 75 years of age with no other health burdens [20].

\section{Pathophysiology of Allergic Rhinitis in Seniors}

Allergic rhinitis is generally caused by immunoglobulin E (IgE)-mediated allergic inflammation in the nasal mucosa $[1,21]$. The acute phase of the allergic reaction develops within minutes of allergen exposure and is primarily caused by histamine and arachidonic acid metabolites (leukotrienes, prostaglandins, and thromboxanes) [1,20]. The late-phase allergic reaction, which occurs within $6-12 \mathrm{~h}$ of allergen exposure, is characterized by an influx of monocytes, $\mathrm{T}$ lymphocytes, basophils, and eosinophils to the nasal mucosa $[1,21,22]$. In elderly patients, this phase may be not noticed by patients due to the very limited or non-existent course [11]. The intermittent nasal symptoms induced by an allergy to pollens or molds might also generate intensive symptoms in elderly patients, although a significant reduction may occur in patients over 75 years of age. The persistent allergic rhinitis symptoms induced by house dust mites, pet dander, and cockroach allergens are frequently indistinguishable from non-allergic rhinitis symptoms in seniors. However, the total and specific IgE levels are increased in the serum, and positive skin prick tests to common inhalant allergens occur in the majority of these patients [2]. In a small subgroup of elderly patients, only local nasal allergic reactions occur without a positive allergy diagnosis. This local allergic rhinitis is diagnosed on the basis of positive nasal provocation tests and typical allergic symptoms [23]. This disease is difficult to identify, particularly in elderly people [24].

The classification of allergic rhinitis according to ARIA is also helpful for seniors [1].

In elderly patients, allergic rhinitis can coexist with nonallergic rhinitis $[25,26]$. Allergy skin testing and/or IgE measurement are used to distinguish between allergic and non-allergic rhinitis [2]. However, these methods have been criticized by some authors [27]. Non-allergic rhinitis 
generally has a milder course, with fewer symptoms of sneezing, nasal pruritus and conjunctivitis and a worse clinical effect after antihistamine drug intake [2]. This type of rhinitis is predominant in patients over 75 years of age. The proportions of allergic rhinitis and non-allergic rhinitis are the same as the proportions reported in younger patients $[2,8]$.

\section{The Diagnosis of Allergic Rhinitis in the Elderly}

The diagnosis of rhinitis in elderly patients is difficult and requires patience. Elderly people are not a homogenous group [21, 22] and include younger patients in their sixties and older patients in their late seventies or eighties. The younger group has more in common with younger patients and shares the nasal mucosa pathophysiology, a "better profile" of health and a better ability to tolerate drugs that affect the metabolism. Older seniors are a completely different group. This group has numerous comorbidities that must be treated, such as arterial hypertension, brain disorders, and arteriosclerosis. After age 75, patients are frequently polymedicated, which often influences the diagnosis and treatment of allergic rhinitis [22]. For example, acetylcholinesterase inhibitors, which are commonly used in elderly patients with cognitive impairment, increase anterior rhinorrhea [1].

Many doctors believe that the diagnosis of allergies in patients over 60 years old is impossible [28, 29]. Elderly patients and their doctors focus on diseases that more typically affect patients over 60 years of age, such as diabetes, ischemic disease, and arterial hypertension, and minimize the problem of allergic rhinitis. Unfortunately, these attitudes frequently lead to a reduced quality of life [28, 30].

Confirmation of an allergic rhinitis diagnosis requires a detailed medical history, clinical otolaryngological (HNO) examination and diagnostic allergy procedures $[3,21]$.

The guidelines differentiate the following identical symptoms of allergic nasal reactions: watery anterior rhinorrhea, paroxysmal sneezing, nasal obstructions, nasal pruritus and the coexistence of conjunctivitis. The presence of a minimum of two of these symptoms suggests allergic rhinitis.

An allergy diagnosis based on the typical procedure of a skin prick test to identify common allergens and the determination of the total serum and specific IgE concentrations should be conducted in older patients [9]. Nasal provocation tests with inhalant allergens and the use of acoustic rhinometry or anterior rhinomanometry are sometimes helpful [24]. Notably, the diagnostic value of the skin prick test and the serum IgE concentration could be decreased in the elderly due to aging of the skin and immune system [9]. Mediaty et al. observed total serum $\mathrm{IgE}$ reductions with age in patients with allergic diseases, but not in elderly patients with atopic dermatitis and an $\operatorname{IgE}$ concentration of $>300 \mathrm{kU} / \mathrm{L}$ [31]. However, opposing opinions suggest that diagnostic procedures for allergies are possible and valuable because the differences between younger and older patients in skin reactivity and IgE concentrations are not evident $[9,23]$. Huss et al. confirmed a positive skin prick test in approximately three-fourths of 75 elderly patients who were allergic to common inhalant allergens [32]. Despite this limitation, King et al. recommended this type of diagnosis in the elderly as a valuable tool when doctors avoided applying the tests to areas of the skin affected by atrophic changes of the photoaging type [33]. In some studies, authors observed a high concentration of total and specific IgE to inhalants and rare food allergens in patients over 60 years of age [9].

An additional diagnostic procedure that allows monitoring of inflammation in the upper airways is the measurement of nitric oxide [34]. Nasal nitric oxide is a good marker of inflammatory diseases of the upper airways, such as allergic rhinitis, particularly when monitoring therapeutic effectiveness. This method is also used in elderly patients [35].

\section{The Treatment of Allergic Rhinitis in Elderly Patients}

No specific therapies exist for allergic rhinitis in the elderly. Treatments include pharmacological treatment, attempts at allergen-specific immunotherapy and a reduction or elimination of allergens from the patients' environments. According to the guidelines, pharmacological treatment includes topical and oral drugs $[1,3]$. The type of drug used should be related to the clinical rhinitis symptoms [1,21]. Intranasal glucocorticosteroids and intranasal and/or oral antihistamine drugs are the first-line therapies $[1,3]$. The main problem with these therapies in the elderly is interactions between drugs or between drugs and diseases, especially in patients over 75 years of age. The risk of adverse events increases exponentially with the number of drugs used in older people [36]. Additionally, this risk is higher in seniors with concomitant liver or renal impairment [37]. Few studies have analyzed drug-drug interactions in general in elderly people, and most of the findings have suggested that drug metabolism is individual and not dependent on age. Accumulation of a drug that inhibits metabolism can also lead to the intensification of adverse events. One of the most important problems is interactions with anticholinergic drugs. Many drugs have antimuscarinic actions, and combining them in therapy can induce many serious adverse reactions, such as delirium episodes and urinary retention [36]. Unfortunately, there are no detailed data about drugs used for allergic rhinitis. 


\subsection{Glucocorticosteroids}

Glucocorticosteroids are used in upper airway diseases including allergic and non-allergic rhinitis, particularly nonallergic rhinitis with eosinophilia syndrome (NARES), acute and chronic rhinosinusitis with and without nasal polyps, and adenoid hypertrophy with or without middle ear disease [37]. This evidence suggests that intranasal corticosteroids produce greater nasal symptom relief than topical antihistamines $\left(\mathrm{H}_{1}\right.$ receptor antagonists) even if no difference in ocular symptoms has been reported [38, 39]. Corticosteroid nasal sprays include beclomethasone dipropionate, budesonide, ciclesonide, flunisolide, fluticasone furoate, fluticasone propionate, triamcinolone acetonide, and mometasone furoate. The design of topically active steroid formulations has provided a much better therapeutic ratio than oral corticosteroids. The pharmacodynamic and pharmacokinetic properties of these agents play important roles in facilitating local anti-inflammatory activity with a low rate of side effects [40, 41]. No data suggest increased side effects after the use of these drugs in older patients [36].

Many studies have confirmed that intranasal steroids are most beneficial in allergic rhinitis in general, due to their antiinflammatory activities $[1,41]$. Steroids are also the recommended front-line treatment in every type of allergic rhinitis (intermittent or perennial) regardless of the severity in patients over 60 years of age [3, 41]. However, few studies have investigated the prolonged use of nasal steroids in elderly people. These drugs are well tolerated by the elderly, with adverse reactions identical to those described in the younger population, such as epistaxis, dryness and a burning sensation in the nose [11]. The prolonged use of these drugs frequently unblocks the nose and improves olfaction. No data are available regarding the role of chronically administered nasal steroids in osteoporosis and diabetes in elderly people; however, these patients should be monitored. Mometasone and ciclesonide have minimal bioavailability and therefore are the safest options; this effect is particularly important in the elderly [33].

Oral glucocorticosteroids are not recommended for the treatment of allergic rhinitis in older patients [21]. Some guidelines permit their use in some cases of severe allergic rhinitis; however, adverse events must be expected because these events are typical with systemic steroid use and may negate the benefits [1]. Adverse events, including osteoporosis, diabetes, and arterial hypertension, after systemic steroid use are very individual but frequent and may not depend on age [42]. Each patient must be individually analyzed for comorbidities.

\subsection{Antihistamines}

Antihistamines are a standard therapy for most types of allergic rhinitis, conjunctivitis and other allergic skin diseases in elderly patients [21]. Most $\mathrm{H}_{1}$ antihistamines have good absorption when administered orally, and the majority of $\mathrm{H}_{1}$ antihistamines reach effective plasma concentration within $3 \mathrm{~h}$ after administration.

Based on our present knowledge, first-generation antihistamines are not recommended for the treatment of allergic rhinitis, especially in older patients, because there is a high risk of adverse reactions in elderly people due to the lack of receptor specificity as well as these drugs being able to cross the blood-brain barrier [43]. Anxiety, confusion, dyskinesis, sedation or sleepiness are common in older patients, as are arrhythmias, urinary disturbances, constipation, hypotension, memory dysfunction, and problems with kinetic coordination that lead to falls [44, 45]. Additionally, first-generation antihistamines frequently interact with other medications [44].

Second-generation antihistamines are effective in elderly patients with allergic rhinitis in drops and oral forms. Their safety is based on a relatively low rate of passage across the blood-brain barrier. Simons observed that the new $\mathrm{H}_{1}$-receptor antagonists cetirizine and loratadine were less likely to cause adverse central nervous system effects than the old $\mathrm{H}_{1}$-antagonists in the elderly [48]. However, no sufficient and precise data showing that these drugs are safe for the elderly are available.

Fexofenadine, cetirizine, loratadine, levocetirizine, desloratadine, bilastine and ebastine are most commonly used in elderly people [21]. These drugs have a high affinity for the $\mathrm{H}_{1}$ receptor and no or weak antagonist activity against the anticholinergic and alfa-adreno receptors [36, 44]. The use of bilastine should be emphasized because it has been proven to have no effect on the cholinergic receptor, which is of great importance for safe application in elderly people [45]. Unfortunately, desloratadine and loratadine have important interactions with the cholinergic receptors and thus are not indicated for patients with symptoms such as dry eye syndrome [46, 47].

Some studies have established the anti-inflammatory effects of second-generation antihistamine drugs. Additionally, their interactions with other drugs are relatively slight [48]. However, the majority of second-generation antihistamines are metabolized by the cytochrome 450 enzyme during their first pass through the liver and thus are not recommended for patients with significant liver dysfunction [48]. Furthermore, for drugs with systemic absorption, impairment of renal or hepatic function may be important. For example, cetirizine is excreted by the kidney, and in patients with renal insufficiency it must be used with caution. No mention is made of this issue, and this is of particular importance because renal insufficiency is frequent in the elderly, even in those with normal serum creatinine. Therefore, patients with renal impairment should take lower daily doses of antihistamines such as 
azelastine, ebastine, desloratadine and cetirizine $[49,50]$. Apart from terfenadine, the remaining drugs in this group are cardiologically safe. However, parallel use of secondgeneration antihistamines (particularly ebastine) with drugs (i.e., ketoconazole, macrolides, quinolones, and cimetidine) that inhibit microsomal enzymes of the liver could stimulate arrhythmias in older patients [51].

Topically applicable antihistamines, such as nasal sprays or eye drops, do not induce systemic adverse reactions or drug interactions [50-52].

Doctors should choose antihistamines for elderly patients through careful consideration of the risk of adverse events based on laboratory renal and liver function tests. The reduction of the daily dose of oral antihistamine should be especially considered in patients over 75 years of age $[49,51]$.

\subsection{Decongestants}

This group of topical or systemic drugs affects nasal blockage, which is a significant problem in affected patients. Therefore, these drugs are unfortunately over used and should not be a first-line therapy or used as a monotherapy, particularly for a prolonged period $[1,21]$. Notably, these drugs may generate many adverse events, such as increased arterial hypertension, headache, arousal, prostatism, and aggravation of glaucoma, and urination, which are particularly burdensome in the elderly [21].

\subsection{Antileukotrienes}

Cysteinyl leukotrienes (CysLTs) are key elements in allergic inflammation. Thus, the antagonism of CysLTs appears to be a potential target for allergic rhinitis management. The combination of antileukotrienes and antihistamines showed a synergistic effect for the treatment of seasonal allergic rhinitis. This combination acts quickly on all nasal symptoms and is well tolerated in older patients [51].

\subsection{Intranasal Anticholinergics}

This treatment is occasionally used in elderly patients with allergic rhinitis and persistent rhinorrhea, with good tolerance and no adverse systemic events, but is more frequently used in non-allergic rhinitis [21, 53].

\subsection{Other Treatment Approaches}

Occasionally, nasal irrigation with isotonic sodium chloride is recommended in situations in which nasal dryness coexists with allergic rhinitis. Irrigation is also beneficial for removing the nasal mucosa [11], which is important in patients with an aging nose and dryness.
Combinations of the previously mentioned drugs are recommended when monotherapy is ineffective. This therapeutic step is the same as described in young patients. The most common drug combination is nasal steroids and nasal or oral antihistamines or a short period of use of nasal decongestants with concomitant use of nasal steroids and/ or nasal antihistamines $[1,3]$.

\subsection{Allergen-Specific Immunotherapy}

Allergen-specific subcutaneous immunotherapy is a safe and effective treatment method, particularly for allergic rhinitis in the general population. Several studies have confirmed this therapy's efficacy in several randomized controlled immunotherapy trials, with a high degree of safety and efficacy. However, although doubts regarding the efficacy of immunotherapy in adults and children remain, no broad data exist for elderly patients. Despite the lack of objective contraindications, specific immunotherapy has not played a significant role in treating elderly patients, possibly because of the lack of evidence of safety in this group. This treatment should be considered only in patients with clinical nasal symptoms associated with a confirmation of an IgE-mediated reaction to a specific inhalant allergen. However, unstable circulatory, neoplasm and autoimmune diseases could be very important contraindications and should be very carefully checked in elderly patients [54-56]. Furthermore, immunotherapy should be administered with caution to patients (especially in seniors) receiving beta-blockers or angiotensin-converting enzyme inhibitors because of the higher risk of anaphylactic shock [56].

Recently, some evidence has indicated that allergenspecific immunotherapy is safe and effective in patients over 60 years of age [57]. A double-blind, placebo-controlled trial with sublingual, allergen-specific immunotherapy to Dermatophagoides pteronyssinus and Dermatophagoides farinae in patients over 60 years of age confirmed its efficacy and safety during 3 years of therapy [58]. A total of 51 subjects in the sublingual allergen-specific immunotherapy group and 57 in the placebo group were monitored for 3 years. The total nasal symptom score decreased by $44 \%$ in the active group and $6 \%$ in the placebo group at the end of the 3 years of therapy. The total medication score decreased significantly by a maximum of $51 \%$ in the active group and decreased significantly in the placebo control group. No adverse systemic reactions were reported during the study. These results were confirmed by a similar study with grass pollen [59].

Despite sublingual immunotherapy, Asero assessed the efficacy of injection immunotherapy for birch and ragweed allergies in patients over 54 years of age [60]. However, this trial was not placebo-controlled [60]. In 2016, the first study with injections for allergen immunotherapy to grass pollen with a double-blind, placebo-controlled protocol 
was conducted. Sixty-two 60- to 75-year-old patients with seasonal allergic rhinitis and a confirmed grass pollen allergy according to a skin prick test, nasal provocation and measurement of serum IgE were included in the study. The patients were individually randomized to the active or placebo groups using a double-blind method. A total of 33 subjects in the subcutaneous immunotherapy (SCIT) group and 29 subjects in the placebo group were monitored for 3 years. The total nasal symptom score decreased by $76 \%$ in the active group and 5\% in the placebo group after 3 years of SCIT. At the end of therapy, the total medication score of the active group decreased significantly by a maximum of $62 \%$. No adverse systemic reactions were reported during therapy [61].

More trials are necessary to determine the degree of effectiveness and safety of immunotherapy in elderly patients. A greater application of immunotherapy in elderly people may allow a reduction in chronic drug treatment, which can lead to polytherapy, typical in the elderly.

However, any prolonged therapy can lead to decreased adherence in elderly people. Moreover, some prolonged therapies may not be acceptable to elderly patients. The following factors influence the degree of adherence in elderly people: mental impairment, decreased vision, problems with swallowing and motor deficiencies [62].

In these cases, doctors should monitor therapy, using recommended tools, such as questionnaires or assessment of the number of used drug packages [62].

\subsection{Allergen Avoidance}

Reducing allergen exposure has significantly influenced allergic nasal improvements. Numerous studies have confirmed this influence, particularly in young patients with an allergy to house dust mites [1, 11]. However, Huss et al. demonstrated that high levels of common home allergens, such as dust mites, cockroaches, cats and dogs, could significantly influence allergic asthma and rhinitis in patients over 80 years of age [32].

\section{Conclusions}

Allergic rhinitis occurs more often than is commonly believed in older people. Aging of the immune system does not reduce the frequency of allergic rhinitis. The diagnosis of allergic disease is similar to the diagnosis in young patients. A great deal of evidence indicates that the management of allergic rhinitis in the elderly should be the same as the management in younger patients. However, all recommended therapies should be administered with caution to seniors with other chronic diseases, polymedication and organ dysfunctions. Cooperation between doctors and senior patients and their families or caregivers in the diagnosis and treatment of any allergic disease is also extremely important.

\section{Compliance with Ethical Standards}

Conflict of interest A Bozek has no conflicts of interest related to this manuscript.

Funding No sources of funding were used to support the writing of this manuscript.

Open Access This article is distributed under the terms of the Creative Commons Attribution-NonCommercial 4.0 International License (http://creativecommons.org/licenses/by-nc/4.0/), which permits any noncommercial use, distribution, and reproduction in any medium, provided you give appropriate credit to the original author(s) and the source, provide a link to the Creative Commons license, and indicate if changes were made.

\section{References}

1. Bousquet J, Khalaev N, Cruz AA, Denburg J, Fokkens WJ, et al. Allergic rhinitis and its Impact on Asthma (ARIA) 2008 update (in collaboration with the World Health Organization, GA(2)LEN and AllerGen). Allergy. 2008;63(Suppl 86):8-160. doi:10.1111/j. 1398-9995.2007.01620.x.

2. Nyenhius S, Mathur SK. Rhinitis in older adults. Curr Allergy@ Asthma Rep. 2013;13:171-7. doi:10.1007/s11882-013-03423.

3. Wallace DV, Dykewicz MS, Bernstein DI, Blessing-Moore J, $\operatorname{Cox} \mathrm{L}$, et al. The diagnosis and management of rhinitis: an updated practice parameter. J Allergy Clin Immunol. 2016;22(2 Suppl):1-84. doi:10.106/j.jaci.2008.06.003.

4. Milgrom $\mathrm{H}$, Huang $\mathrm{H}$. Allergic disorders at a venerable age: a mini review. Gerontology. 2014;60(2):99-107. doi:10.1159/ 000355307.

5. Global Initiative for asthma. Gina report. Global strategy for Asthma Management and Prevention 2008. Available at: http:// www.gina.org. Accessed 15 Jun 2014

6. Bodtger U, Poulsen LK, Linneberg A. Rhinitis symptoms and IgE sensitization as risk factors for development of later allergic rhinitis in adults. Allergy. 2006;61:712-6.

7. Busse PJ. Allergic respiratory disease in the elderly. Am J Med. 2007;120:498-502.

8. Lewandowska-Polak A, Wardzynska A, Kowalski ML. Allergy in the elderly. Alergia Astma Immunol. 2015;20:152-8.

9. Bozek A, Jarzab J. Epidemiology of IgE-dependent allergic diseases in elderly patients in Poland. Am J Rhinol Allergy. 2013;27(5):e140-5. doi:10.2500/ajra.2013.27.3920.

10. Wüthrich B, Schmid-Grendelmeier P, Schindler C, Imboden M, Bircher A, Zemp E, Probst-Hensch N. Prevalence of atopy and respiratory allergic diseases in the elderly SAPALDIA population. Int Arch Allergy Immunol. 2013;162(2):143-8. doi:10.1159/ 000351416

11. Yilmaz Sahin AA, Corey JP. Rhinitis in the elderly. Curr Allergy Asthma Rep. 2006;6:125-31.

12. Ciprandi G, Comite P, Ferrero F, Fontana V, Bruzzone M, Mussap M. Serum allergen-specific, allergic rhinitis severity, and age. Rhinology. 2016;54(3):231-8.

13. Baptis AP, Nyenhius S. Rhinitis in the elderly. Immunol Allergy Clin North Am. 2016;36(2):343-57. 
14. Klimek L. Old, wise and allergic: allergies are no longer solely disease of the grandchildren. Int Arch Allergy Immunol. 2014;163:75-6.

15. Edelstein DR. Aging of the normal nose in adults. Laryngoscope. 1996; 106:1-25.

16. Toppozada H. The human nasal mucosa in the menopause (a histochemical and electron microscopic study). J Laryngol Otol. 1988;102:314-8.

17. Eibenstein A, Fioretti AB, Simaskou MN. Olfactory screening tests in mild cognitive impairment. Neurol Sci. 2005;26:156-60.

18. Ogra LP. Ageing and its possible impact on mucosal immune responses. Ageing Res Rev. 2010;9:101-6.

19. Woodland DL, Blackman MA. Immunity and age: living in the past? Trends Immunol. 2006;27:303-7.

20. Bom AT, Pinot AM. Respir Med. 2009;103:1614-22.

21. Hansen J, Klimek L, Hormann K. Pharmacological management of allergic rhinitis in the elderly. Drugs Aging. 2005;22:289-96.

22. Nathan RA. The burden of allergic rhinitis. Allergy Asthma Proc. 2007;28:3-9.

23. Rondon C, Canto G, Blanca M. Local allergic rhinitis: a new entity, characterization and further studies. Curr Opin Allergy Clin Immunol. 2010;10:1-7.

24. Bozek A, Ignasiak B, Kasperska-Zajac A, Scierski W, Grzanka A, Jarzab J. Local allergic rhinitis in elderly patients. Ann Allergy Asthma Immunol. 2015;114(3):199-202. doi:10.1016/j.anai. 2014.12.013.

25. Hanneuse Y, Delespesse G, Hudson D, De Halleux F, Jacques JM. Influence of aging on IgE-mediated reactions in allergic patients. Clin Allergy. 1978;8:165-74.

26. Settipane RA, Charnock DR. Epidemiology of rhinitis: allergic and nonallergic. Clin Allergy Immunol. 2007;19:23-34.

27. Di Lorenzo G, Pacor ML, Amodio E, Leto-Barone MS, Piana LA, D'Alcamo SA, Ditta V, Martinelli N, Di Bona D. Differences and similarities between allergic and nonallergic rhinitis in a large sample of adult patients with rhinitis symptoms. Int Arch Allergy Immunol. 2011;15:355-61.

28. Ventura MT, Scichilone N, Geraldi M, Patella V, Ridolo E. Management of allergic disease in the elderly: key considerations, recommendations and emerging therapies. Expert Rev Clin Immunol. 2015;11(11):1219-28.

29. Pinto JM, Jeswani S. Rhinitis in the geriatric population. Allergy Asthma Clin Immunol. 2010;6:10-1.

30. Blaiss MS. Quality of life in allergic rhinitis. Ann Allergy Asthma Immunol. 1999;83:449-54.

31. Mediaty A, Neuber K. Total and specific serum IgE, decreases with the age in patients with allergic rhinitis, asthma and insect allergy but not in patients with atopic dermatitis. Immun Aging. 2005;31:9.

32. Huss K, Naumann PL, Mason PJ, et al. Asthma severity, atopic status, allergen exposure and quality of life in elderly persons. Ann Allergy Asthma Immunol. 2001;86:524-30.

33. King MJ, Tamulis T, Lockey RF. Prick puncture skin tests and serum specific IgE as predictors of nasal challenge response to Dermatophagoides pteronyssinus in older adults. Ann Allergy Asthma Immunol. 2008;101:12-7.

34. Sahin G, Klimek L, Mullol J, Hörmann K, Walther LE, Pfaar O. Nitric oxide: a promising methodological approach in airway diseases. Int Arch Allergy Immunol. 2011;156(4):352-61. doi:10. $1159 / 000324678$.

35. Bozek A, Krajewska J, Jarzab J. Nasal nitric oxide and other diagnostic procedures in seasonal allergic rhinitis: elderly vs juvenile patients. Am J Otolaryngol. 2011;32(2):105-8. doi:10. 1016/j.amjoto.2009.11.002.

36. Cusack BJ, Vestal RE. Clinical pharmacology: special considerations in the elderly. In: Calkins E, editor. Practice of geriatric medicine. Davis PF: Ford AB. Philadelphia WB Saunders; 1986. p. 115-34.
37. Austin RJ, Maschera B, Walker A, Fairbairn L, Meldrum E, Farrow SN, et al. Mometasone furoate is a less specific glucocorticoid than fluticasone propionate. EurRespir J. 2002;20:1386-92.

38. Smith CL, Kreutner W. In vitro glucocorticoid receptor binding and transcriptional activation by topically active glucocorticoids. Arzneimittelforschung. 1998;48:956-60.

39. Bielory L, Blaiss M, Fineman SM, Ledford DK, Lieberman P, Simons FE, et al. Concerns about intranasal corticosteroids for over-the-counter use: position statement of the Joint Task Force for the American Academy of Allergy, Asthma and Immunology and the American College of Allergy, Asthma and Immunology. Ann Allergy Asthma Immunol. 2006;96(4):514-25.

40. Can D, Tanaç R, Demir E, Gülen F, Veral A. Is the usage of intranasal glucocorticosteroids alone in allergic rhinitis sufficient? Allergy Asthma Proc. 2006;27(3):248-53.

41. Klimek L, Bachert C. Aktuelle aspekte der nasalen Glukokortikosteroidotherapie. HNO. 2000;48:544-55.

42. Moghadam-Kia S, Werth VP. Prevention and treatment of systemic glucocorticoid side effects. Int $\mathrm{J}$ Dermatol. 2010;49(3):239-48.

43. Leurs R, Church MK, Taglialatela M. H1-antihistamines: inverse agonism, anti-inflammatory actions and cardiac effects. Clin Exp Allergy. 2002;32:489-98.

44. Townley RG. Antiallergic properties of the second-generation H1 antihistamines during the early and late reactions to antigen. J Allergy Clin Immunol. 1992;90:720-5.

45. Carter NJ. Bilastine. Drugs. 2012;72(9):1257-69.

46. Devillier P, Roche N, Faisy C. Clinical pharmacokinetics and pharmacodynamics of desloratadine, fexofenadine and levocetirizine: a comparative review. Clin Pharmacokinet. 2008;47:217-30.

47. Wolff SC, Brubaker K, Navratil T, et al. Evaluation of muscarinic receptor antagonism by antihistamines. Allergy. 2007;62(suppl 83): 138.

48. Simons FER. The antiallergic effects of antihistamines (H1-receptor antagonists). J Allergy Clin Immunol. 1992;90:705-15.

49. Jones AW. Perspectives in drug development and clinical pharmacology: the discovery of histamine $\mathrm{H} 1$ and $\mathrm{H} 2$ antagonists. Clin Pharmacol Drug Dev. 2016;5(1):5-12. doi:10.1002/cpdd. 236.

50. Kaliner MA. H1-antihistamines in the elderly. Clin Allergy Immunol. 2002;17:465-81.

51. McCue J. Safety of antihistamines in the treatment of allergic rhinitis in the elderly. Arch Fam Med. 1996;5:464-84.

52. Yap YG, Camm AJ. The current cardiac safety situation with antihistamines. Clin Exp Allergy 1999;29(Suppl 1):15-24.

53. Ciftci Z, Catli T, Hanci D, Cingi C, Erdogan G. Rhinorrhea in the elderly. Eur Arch Otorhinollaryngol. 2015;272:2587-92.

54. Bousquet J, Lockey R, Malling HJ. Allergen immunotherapy: therapeutic vaccines for allergic diseases. A WHO position paper. J Allergy Clin Immunol. 1998;102:558-62.

55. Matricardi PM, Kuna P, Panetta V, Wahn U, Narkus A. Subcutaneous immunotherapy and pharmacotherapy in seasonal allergic rhinitis: a comparison based on meta-analyses. J Allergy Clin Immunol. 2011;128:791-9.

56. Jutel M, Agache I, Bonini S, Burks AW, Calderon M, Canonica W. International consensus on Allergen Immunotherapy II: mechanisms, standardization, and pharmacoeconomics. J Allergy Clin Immunol. 2016;137(2):358-68. doi:10.1016/j.jaci.2015.12. 1300.

57. Armentia A, Fernandez A, Tapias JA, et al. Immunotherapy with allergic extracts in geriatric patients: evaluation of effectiveness and safety. Allergol Immunopathol. 1995;21:193-6.

58. Bozek A, Ignasiak B, Filipowska B, Jarzab J. House dust mite sublingual immunotherapy: a double-blind, placebo-controlled 
study in elderly patients with allergic rhinitis. Clin Exp Allergy. 2012;43:242-8

59. Bozek A, Kolodziejczyk K, Warkocka-Szoltysek B, Jarzab J. Grass pollen sublingual immunotherapy: a double-blind, placebocontrolled study in elderly patients with seasonal allergic rhinitis. Am J Rhinol Allergy. 2014. doi:10.2500/ajra.2014.28.4091.

60. Asero R. Efficacy of injection immunotherapy with ragweed and birch pollen in elderly patients. Int Arch Allergy Immunol. 2004;135:332-5.
61. Bozek A, Kolodziejczyk K, Krajewska-Wojtys A, Jarzab J. Preseasonal, subcutaneous immunotherapy: a double-blinded, placebo-controlled study in elderly patients with an allergy to grass. Ann Allergy Asthma Immunol. 2016;116(2):156-61. doi:10. 1016/j.anai.2015.12.013.

62. Sergi G, De Rui M, Sarti S, Manzato E. Polypharmacy in the elderly: can comprehensive geriatric assessment reduce inappropriate medication use? Drugs Aging. 2011;28(7):509-18. doi:10.2165/11592010-000000000-00000. 